\title{
Floquet vacuum engineering: Laser-driven chiral soliton lattice in the QCD vacuum
}

\author{
Akihiro Yamada and Naoki Yamamoto \\ Department of Physics, Keio University, Yokohama 223-8522, Japan
}

(Received 27 July 2021; revised 6 September 2021; accepted 13 September 2021; published 28 September 2021)

\begin{abstract}
What happens to the QCD vacuum when a time-periodic circularly polarized laser field with a sufficiently large intensity and frequency is applied? Based on the Floquet formalism for periodically driven systems and the systematic low-energy effective theory of QCD, we show that for a sufficiently large frequency and above a critical intensity, the QCD vacuum is unstable against the chiral soliton lattice of pions; a crystalline structure of topological solitons that spontaneously breaks parity and continuous translational symmetries. Our work would pave the way for novel "Floquet vacuum engineering."
\end{abstract}

DOI: 10.1103/PhysRevD.104.054041

\section{INTRODUCTION}

The quantum vacuum has rich structures, such as quark confinement, chiral symmetry breaking, and Higgs condensation. Exploring its possible phase transitions under extreme conditions, which could have occurred in the early Universe for example, is one of the important questions in high-energy physics. The properties of the vacuum may also be changed by experimental methods, which T. D. Lee called "vacuum engineering" [1]. As one such conventional way, relativistic heavy-ion collisions have been used to study the phase structure of QCD [2,3].

Recently, there have been vigorous activities in the area of condensed matter physics to realize novel quantum states of materials under control using a time-periodic laser field. According to Floquet theory, the nonequilibrium quantum many-body dynamics of a time-dependent Hamiltonian can be described by a time-independent effective Hamiltonian, where quasiequilibrium states can appear emergently. This is the concept of Floquet engineering [4,5].

Although the maximum intensity of the laser is still limited experimentally at the moment, one can ask, as a matter of principle, what happens to the QCD vacuum when a time-periodic circularly polarized laser with a sufficiently large intensity and frequency is applied.

In this paper, we address this question by applying the Floquet theory to the QCD vacuum. ${ }^{1}$ Based on the

\footnotetext{
${ }^{1}$ For previous works on the applications of the Floquet theory to the $\mathcal{N}=2$ supersymmetric QCD based on the AdS/CFT correspondence, see Refs. [6,7].

Published by the American Physical Society under the terms of the Creative Commons Attribution 4.0 International license. Further distribution of this work must maintain attribution to the author(s) and the published article's title, journal citation, and DOI. Funded by SCOAP ${ }^{3}$.
}

systematic low-energy effective theory, we show that the QCD vacuum is unstable against the chiral soliton lattice (CSL) of pions; a crystalline structure of topological solitons with spontaneous breaking of parity and continuous translational symmetries. A similar CSL is known to appear in various systems ranging from condensed matter to highenergy physics, such as chiral magnets [8,9], cholesteric liquid crystals [10], and dense QCD matter in a strong magnetic field [11] or global rotation [12,13]; see also Refs. $[14,15]$ for the CSL in QCD-like theories. This work would provide a new possibility of the "Floquet vacuum engineering."

In this paper, we use the natural units $\hbar=c=1$.

\section{FLOQUET THEORY AND HIGH FREQUENCY EXPANSION}

We first review the Floquet formalism applied to manybody systems. We are interested in the quantum dynamics described by

$$
\mathrm{i} \partial_{t}|\Psi(t)\rangle=H(t)|\Psi(t)\rangle
$$

where $|\Psi(t)\rangle$ is the quantum state at time $t$ and $H(t)=$ $H_{0}+H_{\text {laser }}(t)$ is the total Hamiltonian, with $H_{0}$ being the time-independent part of the Hamiltonian and $H_{\text {laser }}(t)$ the laser-induced time-dependent Hamiltonian satisfying $H_{\text {laser }}(t+T)=H_{\text {laser }}(t)$. Here, the periodicity $T$ is related to the driving frequency $\Omega$ via $T=2 \pi / \Omega$.

The Floquet theorem, which is the temporal version of the Bloch theorem for spatially periodic crystals, states that the solution of the time-periodic Hamiltonian above can be expressed as

$$
|\Psi(t)\rangle=\mathrm{e}^{-\mathrm{i} \varepsilon t}|\Phi(t)\rangle, \quad|\Phi(t+T)\rangle=|\Phi(t)\rangle,
$$

where $\varepsilon$ is the Floquet quasienergy. 
As the total Hamiltonian is periodic, $H(t+T)=H(t)$, we can perform the discrete Fourier transform as

$$
H(t)=\sum_{n} \mathrm{e}^{-\mathrm{i} n \Omega t} H_{n}, \quad|\Phi(t)\rangle=\sum_{n} \mathrm{e}^{-\mathrm{i} n \Omega t}\left|\Phi_{n}\right\rangle,
$$

with $n$ an integer, and then Eq. (1) can be mapped to a timeindependent eigenvalue problem [16,17],

$$
\sum_{m}\left(H_{n-m}-m \Omega \delta_{m n}\right)\left|\Phi_{m}\right\rangle=\varepsilon\left|\Phi_{n}\right\rangle .
$$

This eigenvalue problem may be solved by a perturbative expansion in terms of $1 / \Omega$ by assuming that the time scale of interest is much larger than $T$. The resulting static effective Hamiltonian up to the first order in $1 / \Omega$ is $[18,19]$

$$
\begin{aligned}
& H_{\mathrm{eff}}=H_{\mathrm{eff}}^{(0)}+H_{\mathrm{eff}}^{(1)}+O\left(\frac{1}{\Omega^{2}}\right), \\
& H_{\mathrm{eff}}^{(0)}=H_{0}, \quad H_{\mathrm{eff}}^{(1)}=\frac{1}{\Omega} \sum_{n>0} \frac{\left[H_{-n}, H_{n}\right]}{n},
\end{aligned}
$$

where $H_{\text {eff }}^{(n)}$ denotes the $n$th order effective Hamiltonian in the expansion of $1 / \Omega$.

Note that this truncated effective Hamiltonian describes the transient dynamics for certain long but finite time scale [20,21]. In this sense, the "ground state" that we will discuss based on this effective Hamiltonian should be regarded as a quasiequilibrium state.

\section{APPLICATION TO DIRAC FERMIONS AND CHERN-SIMONS CURRENT}

Let us first apply the Floquet formalism above to generic Dirac fermions (with charge $e$ ) coupled to the laser field in three spatial dimensions. ${ }^{2}$ In the following, we adopt the Coulomb gauge $\boldsymbol{\nabla} \cdot \boldsymbol{A}=0$. The time-dependent coupling to the laser field is given by

$$
H_{\text {laser }}(t)=-e \int \mathrm{d}^{3} \boldsymbol{x} \psi^{\dagger} \gamma^{0} \boldsymbol{\gamma} \cdot \boldsymbol{A} \psi,
$$

where $\gamma^{\mu}(\mu=0,1,2,3)$ are the gamma matrices and $\boldsymbol{A}=\boldsymbol{A}(t, \boldsymbol{x})$ is the time-periodic gauge field, which we take to be

$$
\boldsymbol{A}(t, \boldsymbol{x})=\boldsymbol{A}^{+}(\boldsymbol{x}) \mathrm{e}^{-\mathrm{i} \Omega t}+\boldsymbol{A}^{-}(\boldsymbol{x}) \mathrm{e}^{\mathrm{i} \Omega t}
$$

\footnotetext{
${ }^{2}$ See also Ref. [22] for the application of the Floquet theory to Dirac fermions. Here, we demonstrate the direct relevance of the Chern-Simons current in the Floquet effective Hamiltonian for a more generic position-dependent gauge field, which is new to the best of our knowledge.
}

Inserting $H_{ \pm 1}$ into Eq. (5) and using the identity

$$
2 \mathrm{i} \Omega \boldsymbol{A}^{+} \times \boldsymbol{A}^{-}=\boldsymbol{E} \times \boldsymbol{A},
$$

we have

$$
H_{\mathrm{eff}}^{(1)}=-\frac{e^{2}}{\Omega^{2}} \int \mathrm{d}^{3} \boldsymbol{x}(\boldsymbol{E} \times \boldsymbol{A}) \cdot \dot{j}^{5},
$$

where $j^{5 \mu}=\bar{\psi} \gamma^{\mu} \gamma^{5} \psi$ is the axial current. By using the Chern-Simons current defined by $j_{\mathrm{CS}}^{\mu}=\frac{1}{2} \epsilon^{\mu \nu \alpha \beta} A_{\nu} F_{\alpha \beta}$, Eq. (9) can be written as the form of the current-current interaction

$$
H_{\mathrm{eff}}^{(1)}=-\frac{e^{2}}{\Omega^{2}} \int \mathrm{d}^{3} x j_{\mathrm{CS}} \cdot j^{5}
$$

Recall that the axial current $j^{5 \mu}$ carries the helicity of fermions while the Chern-Simons current $j_{\text {CS }}^{\mu}$ carries the helicity of electromagnetic fields. Hence, through this interaction term, the helicity of the laser induces the helicity of matter sector composed of Dirac fermions.

For definiteness, below we take

$$
\boldsymbol{A}(t, \boldsymbol{x})=\frac{F}{\Omega}(\cos \Omega(t-z), \lambda \sin \Omega(t-z), 0),
$$

where $F$ is the amplitude of the electromagnetic fields (see below) and $\lambda= \pm 1$ corresponds to the helicity of the gauge field. The corresponding $\boldsymbol{A}^{ \pm}$read

$$
\boldsymbol{A}^{+}(\boldsymbol{x})=\frac{F}{2 \Omega} \mathrm{e}^{\mathrm{i} \Omega z} \boldsymbol{e}_{ \pm}, \quad \boldsymbol{A}^{-}(\boldsymbol{x})=\frac{F}{2 \Omega} \mathrm{e}^{-\mathrm{i} \Omega z} \boldsymbol{e}_{\mp},
$$

for $\lambda= \pm 1$, respectively, where $\boldsymbol{e}_{ \pm}=\boldsymbol{e}_{x} \pm \mathrm{i} \boldsymbol{e}_{y}$ are the helicity basis with $\boldsymbol{e}_{x, y, z}$ being the unit vectors in the $x$, $y, z$ directions. Note that the gauge field above satisfies the transversality condition $\boldsymbol{e}_{z} \cdot \boldsymbol{A}=0$.

In this case, the electromagnetic components of the laser field are

$$
\begin{aligned}
\boldsymbol{E}(t, \boldsymbol{x}) & =F(\sin \Omega(t-z),-\lambda \cos \Omega(t-z), 0), \\
\boldsymbol{B}(t, \boldsymbol{x}) & =F(\lambda \cos \Omega(t-z), \sin \Omega(t-z), 0),
\end{aligned}
$$

and the Chern-Simons current is

$$
j_{\mathrm{CS}}=\lambda \frac{F^{2}}{\Omega} \boldsymbol{e}_{z}
$$

Then, the effective Hamiltonian density (10) becomes 


$$
\mathcal{H}_{\text {eff }}^{(1)}=-\lambda \frac{e^{2} F^{2}}{\Omega^{3}} j_{z}^{5} .
$$

Alternatively, one might take a position-independent gauge field

$$
\boldsymbol{A}(t)=\frac{F}{\Omega}(\cos \Omega t, \lambda \sin \Omega t, 0),
$$

which also leads to the same Chern-Simons current and the effective Hamiltonian density as above. However, the choice of the position-dependent gauge field (11), which involves not only the rotating electric field but also the rotating magnetic field, is essential in order not to cause the Schwinger effect [23], as we will discuss later.

\section{LOW-ENERGY EFFECTIVE THEORY OF QCD IN THE LASER FIELD}

We now consider the vacuum of two-flavor QCD in the laser field. We are interested in the low-energy regime, whose dynamical degrees of freedom are pions-the (pseudo) Nambu-Goldstone (NG) modes associated with chiral symmetry breaking. The QCD dynamics in this regime is described by the effective field theory for pions, called the chiral perturbation theory (see, e.g., Ref. [24] for a review). Since the generally strong and rapidly changing external electromagnetic field explicitly breaks the (approximate) coset symmetry $\left[\mathrm{SU}(2)_{\mathrm{R}} \times \mathrm{SU}(2)_{\mathrm{L}}\right] / \mathrm{SU}(2)_{\mathrm{V}}$ down to $\mathrm{U}(1)_{\mathrm{A}}^{I_{3}}$, we will focus on the light and slow $\pi_{0}$ sector. We will thus need to consider the original timedependent part of the Hamiltonian for $\pi_{0}$ augmented by the laser-driven static Floquet Hamiltonian for $\pi_{0}$.

Following the general idea of the low-energy effective field theory, we perform a systematic expansion in terms of $\epsilon_{1} \equiv p / \Lambda$ and $\epsilon_{2} \equiv m_{\pi} / \Lambda$, where $p$ is the typical energy scale of pions, $\Lambda=4 \pi f_{\pi}$ is the energy scale for chiral symmetry breaking (with $f_{\pi}$ being the pion decay constant), and $m_{\pi}$ is the pion mass in the QCD vacuum. As already described above, we also need to perform the expansion in terms of $\epsilon_{3} \equiv p / \Omega$. In addition, one may set up certain counting for the terms involving $e F$. As $e F$ appears only through the combination $e^{2} F^{2} / \Omega^{3}$ [the coefficient of Eq. (16)] in the Floquet Hamiltonian at leading order in $1 / \Omega$, we introduce $\epsilon_{4} \equiv e^{2} F^{2} /\left(\Omega^{3} \Lambda\right)$ as another small parameter. To be specific, we adopt the counting scheme that they are all comparable, $\epsilon_{1,2,3,4} \sim \epsilon$, and we write down the effective theory up to $O\left(\epsilon^{2}\right)$ in this paper.

We first consider QCD without the coupling to the laser field. The effective theory up to $O\left(\epsilon^{2}\right)$ is

$$
\mathcal{L}_{\mathrm{EFT}}=\frac{f_{\pi}^{2}}{4}\left[\operatorname{tr}\left(\partial_{\mu} \Sigma \partial^{\mu} \Sigma^{\dagger}\right)+m_{\pi}^{2} \operatorname{tr}\left(\Sigma+\Sigma^{\dagger}\right)\right],
$$

where the phases of $\Sigma \in \mathrm{SU}(2)$ describe the pion fields and $\operatorname{tr}(\ldots)$ denotes the trace over the flavor space.

Let us then consider the coupling of QCD to the laser field. In this case, the current-current interaction (10) needs to be extended to include the color and flavor degrees of freedom as

$$
\begin{aligned}
\mathcal{H}_{\text {Floquet }} & =-\frac{e^{2}}{\Omega^{2}} \sum_{\mathrm{f}=\mathrm{u}, \mathrm{d}} Q_{\mathrm{f}}^{2} \boldsymbol{j}_{\mathrm{CS}} \cdot j_{\mathrm{f}}^{5} \\
& =-\frac{e^{2}}{\Omega^{2}} \boldsymbol{j}_{\mathrm{CS}} \cdot\left(\frac{1}{3} j_{3}^{5}+\frac{5}{18} j^{5}\right) .
\end{aligned}
$$

Here, $\left(Q_{\mathrm{u}}, Q_{\mathrm{d}}\right)=(2 / 3,-1 / 3)$ is the quark charge, $j_{\mathrm{u}}^{5 \mu}=$ $\overline{\mathrm{u}} \gamma^{\mu} \gamma^{5} \mathrm{u}$ and $j_{\mathrm{d}}^{5 \mu}=\overline{\mathrm{d}} \gamma^{\mu} \gamma^{5} \mathrm{~d}$ are axial currents for up and down quarks, and $j_{a}^{5 \mu}=\bar{q} \gamma^{\mu} \gamma^{5} \frac{\tau_{a}}{2} q$ and $j^{5 \mu}=\bar{q} \gamma^{\mu} \gamma^{5} q$ are flavortriplet and singlet axial currents, respectively, with $\tau_{a}$ $(a=1,2,3)$ being the $\mathrm{SU}(2)$ generators satisfying the normalization $\operatorname{tr}\left(\tau_{a} \tau_{b}\right)=2 \delta_{a b}$.

At low energy, the axial current $j_{a}^{5 \mu}$ is carried by pions $\pi_{a}$ and can be expressed in terms of $\Sigma$ as

$$
j_{a}^{5 \mu}=-\mathrm{i} \frac{f_{\pi}^{2}}{4} \operatorname{tr}\left[\tau_{a}\left(\Sigma \partial^{\mu} \Sigma^{\dagger}+\partial^{\mu} \Sigma^{\dagger} \Sigma\right)\right] .
$$

Since only the $a=3$ component is relevant for the Floquet Hamiltonian (19), we set $\Sigma=\mathrm{e}^{\mathrm{i} \tau_{3} \pi_{0}}$, for which $j_{3}^{5 \mu}=-f_{\pi}^{2} \partial^{\mu} \pi_{0} .{ }^{3}$ By requiring the UV-IR matching for the term (19), the Floquet Hamiltonian density in terms of $\pi_{0}$ becomes

$$
\mathcal{H}_{\text {Floquet }}=-\lambda \frac{e^{2} F^{2} f_{\pi}^{2}}{3 \Omega^{3}} \partial_{z} \pi_{0},
$$

which is also $O\left(\epsilon^{2}\right)$ in our counting scheme.

The Floquet Hamiltonian (21) leads to a nontrivial consequence in the $\pi_{0}$ sector, on which we focus below. The total low-energy effective Hamiltonian density is then given by $\mathcal{H}_{\text {total }}=\mathcal{H}_{\pi_{0}}+\mathcal{H}_{\text {Floquet }}$, where

$$
\mathcal{H}_{\pi_{0}}=\frac{f_{\pi}^{2}}{2}\left(\nabla \pi_{0}\right)^{2}+m_{\pi}^{2} f_{\pi}^{2}\left(1-\cos \pi_{0}\right) .
$$

Here, we also included the offset $m_{\pi}^{2} f_{\pi}^{2}$ such that $\left\langle\mathcal{H}_{\pi_{0}}\right\rangle=0$ for $\left\langle\pi_{0}\right\rangle=0$, with $\langle\ldots\rangle$ being the vacuum expectation value. Note that the Wess-Zumino-Witten term of the form $\pi_{0} \boldsymbol{E} \cdot \boldsymbol{B}[25,26]$ is irrelevant here since $\boldsymbol{E} \perp \boldsymbol{B}$ in the present setup.

The total Hamiltonian density is trivially minimized in the $x$ and $y$ directions by taking $\left\langle\partial_{x} \pi_{0}\right\rangle=\left\langle\partial_{y} \pi_{0}\right\rangle=0$. Then, the remaining Hamiltonian density in the $z$ direction is

\footnotetext{
${ }^{3}$ We ignore the contribution of the flavor-singlet axial current $j^{5 \mu}$ carried by the heavy $\eta$ meson.
} 
$\mathcal{H}_{\text {total }}^{z}=\frac{f_{\pi}^{2}}{2}\left(\partial_{z} \pi_{0}\right)^{2}-\lambda \frac{e^{2} F^{2} f_{\pi}^{2}}{3 \Omega^{3}} \partial_{z} \pi_{0}+m_{\pi}^{2} f_{\pi}^{2}\left(1-\cos \pi_{0}\right)$.

We observe that the Hamiltonian (23) has mathematically the same form as those appearing in chiral magnets $[8,9]$, cholesteric liquid crystals [10], and QCD at finite baryon chemical potential in a background magnetic field [11] or global rotation [12,13]; through the Floquet engineering, this result provides a new universality between high-energy physics and condensed-matter physics. In particular, the Floquet effective Hamiltonian (21) for pions has the same form as the Dzyaloshinskii-Moriya interaction for magnons in chiral magnets $[8,9]$ and the Wess-ZuminoWitten term for pions in QCD matter at finite density $[27,28]$. By utilizing the results there, we can also find the analytic solutions for the ground state of our Floquet-QCD system. Below we consider $\lambda=1$ as an example.

\section{CHIRAL HELIX AND HELICITY TRANSFER}

Let us start with QCD in the chiral limit where $m_{\pi}=0$. In this case, the Hamiltonian density (23) is minimized when

$$
\left\langle\pi_{0}\right\rangle=\frac{e^{2} F^{2}}{3 \Omega^{3}} z,
$$

at which the minimum is given by

$$
\left\langle\mathcal{H}_{\text {total }}^{z}\right\rangle=-\frac{1}{2}\left(\frac{e^{2} F^{2} f_{\pi}}{3 \Omega^{3}}\right)^{2}<0 .
$$

This shows that the QCD vacuum is unstable for an infinitesimally small amplitude $F$ against the formation of the helical structure of $\pi_{0}$, given by Eq. (24), which spontaneously breaks the parity symmetry. This has the natural interpretation that the QCD vacuum is turned into the helical ground state by the helicity of the laser field.

In fact, in this case we have the following helicity transfer relation

$$
\frac{1}{f_{\pi}^{2}} j_{3}^{5}=\frac{e^{2}}{3 \Omega^{2}} j_{\mathrm{CS}}
$$

Note that in usual relativistic quantum field theories, helicity transfer from the gauge sector to the matter sector occurs through the effects related to the chiral anomaly [29]. In the present case, however, this new type of helicity transfer can occur owing to the Floquet effective interaction (10).

\section{CHIRAL SOLITON LATTICE AND CRITICAL INTENSITY}

In the case of QCD with finite quark masses, the equation of motion for the Hamiltonian density (23) is given by

$$
\partial_{z}^{2} \pi_{0}=m_{\pi}^{2} \sin \pi_{0}
$$

This is the same equation of motion for a single pendulum and can be analytically solved using the Jacobi elliptic function (see also Refs. [9,11]),

$$
\cos \frac{\pi_{0}(\bar{z})}{2}=\operatorname{sn}(\bar{z}, k)
$$

where $\bar{z} \equiv m_{\pi} z / k$ is a dimensionless coordinate and $k$ $(0 \leq k \leq 1)$ is the elliptic modulus.

This solution shows that $\pi_{0}(\bar{z})$ changes from $2(n-1) \pi$ to $2 n \pi$ for $(2 n-1) K(k) \leq \bar{z} \leq(2 n+1) K(k)$ with $n$ being an integer and $K(k)$ the complete elliptic integral of the first kind. Hence, the period of the crystalline structure is

$$
\ell=\frac{2 k K(k)}{m_{\pi}}
$$

We can also show that each lattice carries the axial current as a topological charge. In fact, we have

$$
\int_{(2 n-1) K}^{(2 n+1) K} \mathrm{~d} \bar{z} j_{3}^{5 z}(\bar{z})=2 \pi f_{\pi}^{2}
$$

which is topologically quantized independently of the detailed profile of $\pi_{0}(z)$.

In summary, this ground-state solution breaks parity and continuous translational symmetries and has the topological charge; thus, this is the CSL phase similar to those in other systems [8-13] (see also Ref. [30] for the laser-driven CSL in multiferroic magnets). It is interesting to note that although the microscopic Hamiltonians are quite different between QCD and multiferroics, the same type of the ground state is naturally realized in the presence of the circularly polarized laser.

Let us now derive the critical intensity for the realization of CSL in the QCD vacuum. The energy of each lattice per unit area in the $x y$ plane is given by

$$
\begin{aligned}
\frac{\mathcal{E}_{\text {lattice }}}{S} & =4 m_{\pi} f_{\pi}^{2}\left[\frac{2 E(k)}{k}+\left(k-\frac{1}{k}\right) K(k)\right]-\frac{2 \pi e^{2} F^{2} f_{\pi}^{2}}{3 \Omega^{3}} \\
& \equiv G(k),
\end{aligned}
$$

where $E(k)$ is the complete elliptic integral of the second kind. The total energy at length $L$ in the $z$ direction is then ${ }^{4}$

\footnotetext{
${ }^{4}$ Generally, the quasienergy in Floquet systems is defined modulo multiples of $\Omega$, and possible Floquet-Umklapp scattering processes may violate the energy conservation. In the regime we consider, however, the energy transfer of the physical degrees of freedom is much smaller than $\Omega$, and such Floquet-Umklapp processes do not occur. Hence, the total energy here is well defined.
} 


$$
\mathcal{E}_{\text {total }}=\frac{V}{\ell} G(k)
$$

where $V=L S$ is the volume of the system.

Minimizing $\mathcal{E}_{\text {total }}$ at fixed $V$ with respect to $k$ yields

$$
\frac{E(k)}{k}=\frac{\pi e^{2} F^{2}}{12 m_{\pi} \Omega^{3}} .
$$

From the inequality $E(k) / k \geq 1$ for $0 \leq k \leq 1$, we find the critical amplitude for the CSL solution,

$$
F \geq F_{\mathrm{CSL}} \equiv \sqrt{\frac{12 m_{\pi} \Omega^{3}}{\pi e^{2}}}
$$

and the corresponding critical intensity $I_{\mathrm{CSL}}=F_{\mathrm{CSL}}^{2}$. This is our main result. In the chiral limit, it reduces to $F_{\mathrm{CSL}}=0$, as is consistent with the result of the chiral helix above. For $F=F_{\mathrm{CSL}}$, the parameter $\epsilon_{4}$ satisfies

$$
\epsilon_{4}=\frac{12 m_{\pi}}{\pi \Lambda} \ll 1
$$

which confirms the consistency of our analysis.

One might think that the vacuum may also be potentially unstable against the pair production of charged pions $\pi_{ \pm}$ due to the Schwinger effect [23] in the confined phase. For the specific choice of the gauge field (11), however, one can argue on physical grounds that the Schwinger effect does not occur. For $\pi_{ \pm}$(with the charge $q= \pm e$ ) initially at rest, the classical trajectories after turning on this laser field are given by [31]

$$
\begin{aligned}
& x(t)=-\frac{q F}{m_{\pi} \Omega^{2}} \sin \left(\frac{\Omega t}{\gamma}\right), \\
& y(t)=\frac{q F}{m_{\pi} \Omega^{2}} \cos \left(\frac{\Omega t}{\gamma}\right), \\
& z(t)=\frac{(q F)^{2} t}{(q F)^{2}+2\left(m_{\pi} \Omega\right)^{2}},
\end{aligned}
$$

where

$$
\gamma=1+\frac{1}{2}\left(\frac{q F}{m_{\pi} \Omega}\right)^{2} .
$$

Hence, in this case the laser field does not tear the pair of $\pi_{ \pm}$apart, as a static magnetic field does not [32]. This should be contrasted with the position-independent gauge field (17), for which the rotating electric field can break the pair of $\pi_{ \pm}$apart, triggering the Schwinger effect $[7,33]$.

\section{APPLICATION TO WEYL/DIRAC SEMIMETALS}

The argument and results so far should also be applied to Weyl/Dirac semimetals with chiral symmetry breaking (see, e.g., Ref. [34]). In such a system, there is a gapless NG mode associated with U(1) chiral symmetry breaking, which may be regarded as an axion field. By repeating the same argument as above, we can construct a low-energy effective theory for $\theta$ under the laser field (11) well below the energy gap as

$$
\mathcal{H}_{\theta}=\frac{f^{2}}{2}(\boldsymbol{\nabla} \theta)^{2}-\lambda \frac{e^{2} F^{2} f^{2}}{\Omega^{3}} \partial_{z} \theta
$$

Here, $f$ is some parameter which cannot be fixed by the symmetry argument alone (similar to $f_{\pi}$ in the chiral perturbation theory). We can then show that the original insulating phase is unstable against the formation of the chiral helix of $\theta$,

$$
\langle\theta\rangle=\lambda \frac{e^{2} F^{2}}{\Omega^{3}} z
$$

for $F>0$.

The electromagnetic properties of such a chiral helix phase was previously studied in a different context in Ref. [35]. If we further turn on an external electric field $\boldsymbol{E}_{\mathrm{ex}}$, it induces the anomalous Hall effect,

$$
j_{\mathrm{AHE}}=\frac{e^{2}}{4 \pi^{2}}\langle\nabla \theta\rangle \times \boldsymbol{E}_{\mathrm{ex}}=\lambda \frac{e^{4} F^{2}}{4 \pi^{2} \Omega^{3}} \boldsymbol{e}_{z} \times \boldsymbol{E}_{\mathrm{ex}} .
$$

Also, the dispersion relation of photons in this phase is modified: one of the helicity states acquires an energy gap $\Delta$ while the other has a quadratic dispersion, the latter of which can be regarded as the so-called type-B NG mode of the one-form symmetry [35,36]. As a consequence, the chiral helix behaves as a polarizer which can transmit electromagnetic waves with only one type of helicity below the gap $\Delta$ (see also Ref. [37]). These predictions may in principle be tested in tabletop experiments.

\section{CONCLUSION}

In this paper, we have shown that there exists a previously unknown region of laser intensity characterized by the pion mass, where the QCD vacuum is altered to a novel state of matter, the pion chiral soliton lattice, by the new mechanism. For $\Omega \sim \Lambda \sim 1 \mathrm{GeV}$ (corresponding to a yoctosecond laser) with $m_{\pi} \sim 0.1 \mathrm{GeV}$, we obtain $F_{\mathrm{CSL}} \sim(1 \mathrm{GeV})^{2}{ }^{5}$. This is above the critical intensity of the Schwinger mechanism, characterized by the electron

\footnotetext{
${ }^{5}$ Note that this is still within the applicability of the effective theory in that the parameter $\epsilon_{4}$ in Eq. (35) is sufficiently small.
} 
mass, where the QED vacuum becomes unstable due to electron-positron pair production. Therefore, the critical laser intensity of the Schwinger mechanism, which has not been experimentally realized so far, is not the ultimate regime of the quantum vacuum physics, and it would be interesting to further aim for a laser intensity beyond it in the future.

\section{ACKNOWLEDGMENTS}

We thank Koichi Hattori for discussions on the Schwinger effect. This work was supported by the Keio Institute of Pure and Applied Sciences (KiPAS) project at Keio University and JSPS KAKENHI Grant No. 19K03852.
[1] T. D. Lee, Particle Physics and Introduction to Field Theory (Harwood Academic, London, 1988).

[2] M. A. Stephanov, QCD phase diagram and the critical point, Prog. Theor. Phys. Suppl. 153, 139 (2004).

[3] K. Fukushima and T. Hatsuda, The phase diagram of dense QCD, Rep. Prog. Phys. 74, 014001 (2011).

[4] M. Bukov, L. D'Alessio, and A. Polkovnikov, Universal high-frequency behavior of periodically driven systems: From dynamical stabilization to floquet engineering, Adv. Phys. 64, 139 (2015).

[5] T. Oka and S. Kitamura, Floquet engineering of quantum materials, Annu. Rev. Condens. Matter Phys. 10, 387 (2019).

[6] K. Hashimoto, S. Kinoshita, K. Murata, and T. Oka, Holographic Floquet states I: A strongly coupled Weyl semimetal, J. High Energy Phys. 05 (2017) 127.

[7] S. Kinoshita, K. Murata, and T. Oka, Holographic Floquet states II: Floquet condensation of vector mesons in nonequilibrium phase diagram, J. High Energy Phys. 06 (2018) 096.

[8] I. E. Dzyaloshinsky, Theory of helicoidal structures in antiferromagnets. I. Nonmetals, Sov. Phys. JETP 19, 960 (1964).

[9] J.-i. Kishine and A. S. Ovchinnikov, Theory of monoaxial chiral helimagnet, in Solid State Physics (Elsevier, New York, 2015), Vol. 66, pp. 1-130.

[10] P. G. De Gennes, Calcul de la distorsion d'une structure cholesterique par un champ magnetique, Solid State Commun. 6, 163 (1968).

[11] T. Brauner and N. Yamamoto, Chiral soliton lattice and charged pion condensation in strong magnetic fields, J. High Energy Phys. 04 (2017) 132.

[12] X.-G. Huang, K. Nishimura, and N. Yamamoto, Anomalous effects of dense matter under rotation, J. High Energy Phys. 02 (2018) 069.

[13] K. Nishimura and N. Yamamoto, Topological term, QCD anomaly, and the $\eta^{\prime}$ chiral soliton lattice in rotating baryonic matter, J. High Energy Phys. 07 (2020) 196.

[14] T. Brauner, G. Filios, and H. Kolešová, Anomaly-Induced Inhomogeneous Phase in Quark Matter without the Sign Problem, Phys. Rev. Lett. 123, 012001 (2019).

[15] T. Brauner, G. Filios, and H. Kolešová, Chiral soliton lattice in QCD-like theories, J. High Energy Phys. 12 (2019) 029.

[16] J. H. Shirley, Solution of the schrödinger equation with a hamiltonian periodic in time, Phys. Rev. B 138, B979 (1965).
[17] H. Sambe, Steady states and quasienergies of a quantummechanical system in an oscillating field, Phys. Rev. A 7, 2203 (1973).

[18] E.S. Mananga and T. Charpentier, Introduction of the floquet-magnus expansion in solid-state nuclear magnetic resonance spectroscopy, J. Chem. Phys. 135, 044109 (2011).

[19] N. Goldman and J. Dalibard, Periodically-Driven Quantum Systems: Effective Hamiltonians and Engineered Gauge Fields, Phys. Rev. X 4, 031027 (2014); Erratum, Phys. Rev. X 5, 029902 (2015).

[20] T. Kuwahara, T. Mori, and K. Saito, Floquet-magnus theory and generic transient dynamics in periodically driven manybody quantum systems, Ann. Phys. (Amsterdam) 367, 96 (2016).

[21] T. Mori, T. Kuwahara, and K. Saito, Rigorous Bound on Energy Absorption and Generic Relaxation in Periodically Driven Quantum Systems, Phys. Rev. Lett. 116, 120401 (2016).

[22] S. Ebihara, K. Fukushima, and T. Oka, Chiral pumping effect induced by rotating electric fields, Phys. Rev. B 93, 155107 (2016).

[23] J. S. Schwinger, On gauge invariance and vacuum polarization, Phys. Rev. 82, 664 (1951).

[24] S. Scherer, Introduction to chiral perturbation theory, Adv. Nucl. Phys. 27, 277 (2003).

[25] J. Wess and B. Zumino, Consequences of anomalous Ward identities, Phys. Lett. 37B, 95 (1971).

[26] E. Witten, Global aspects of current algebra, Nucl. Phys. B223, 422 (1983).

[27] D. T. Son and A. R. Zhitnitsky, Quantum anomalies in dense matter, Phys. Rev. D 70, 074018 (2004).

[28] D. T. Son and M. A. Stephanov, Axial anomaly and magnetism of nuclear and quark matter, Phys. Rev. D 77, 014021 (2008).

[29] N. Yamamoto, Chiral transport of neutrinos in supernovae: Neutrino-induced fluid helicity and helical plasma instability, Phys. Rev. D 93, 065017 (2016).

[30] M. Sato, S. Takayoshi, and T. Oka, Laser-Driven Multiferroics and Ultrafast Spin Current Generation, Phys. Rev. Lett. 117, 147202 (2016).

[31] J. Meyer-ter Vehn, A. Pukhov, and Zh.-M. Sheng, Relativistic laser plasma interaction, in Atoms, Solids, and Plasmas in Super-Intense Laser Fields (Springer, New York, 2001), pp. 167-192.

[32] G. V. Dunne, Heisenberg-euler effective lagrangians: Basics and extensions, in From Fields to Strings: Circumnavigating 
Theoretical Physics. Ian Kogan Memorial Collection (3 Volume Set), edited by M. Shifman, A. Vainshtein, and J. Wheater (World Scientific Publishing, Singapore, 2005).

[33] S. Takayoshi, J. Wu, and T. Oka, New aspects of nonadiabatic geometric effects-Application to twisted schwinger effect in dirac and Weyl fermions, arXiv:2005.01755.

[34] Z. Wang and S.-C. Zhang, Chiral anomaly, charge density waves, and axion strings from Weyl semimetals, Phys. Rev. B 87, 161107 (2013).
[35] N. Yamamoto, Axion electrodynamics and nonrelativistic photons in nuclear and quark matter, Phys. Rev. D 93, 085036 (2016).

[36] Y. Hidaka, Y. Hirono, and R. Yokokura, Counting NambuGoldstone Modes of Higher-Form Global Symmetries, Phys. Rev. Lett. 126, 071601 (2021).

[37] S. Ozaki and N. Yamamoto, Axion crystals, J. High Energy Phys. 08 (2017) 098. 